This item was submitted to Loughborough's Research Repository by the author.

Items in Figshare are protected by copyright, with all rights reserved, unless otherwise indicated.

\title{
Learning industrial robot force/torque compensation: A comparison of support vector and random forests regression
}

PLEASE CITE THE PUBLISHED VERSION

http://dx.doi.org/10.2316/P.2016.847-002

\section{PUBLISHER}

(c) Acta Press

\section{VERSION}

AM (Accepted Manuscript)

\section{PUBLISHER STATEMENT}

This work is made available according to the conditions of the Creative Commons Attribution-NonCommercialNoDerivatives 4.0 International (CC BY-NC-ND 4.0) licence. Full details of this licence are available at: https://creativecommons.org/licenses/by-nc-nd/4.0/

\section{LICENCE}

CC BY-NC-ND 4.0

\section{REPOSITORY RECORD}

Al-Yacoub, Ali, Sara Sharifzadeh, Niels Lohse, Zahid Usman, Yee M. Goh, and Michael R. Jackson. 2019. "Learning Industrial Robot Force/torque Compensation: A Comparison of Support Vector and Random Forests Regression”. figshare. https://hdl.handle.net/2134/23638. 


\title{
LEARNING INDUSTRIAL ROBOT FORCE/TORQUE COMPENSATION: A COMPARISON OF SUPPORT VECTOR AND RANDOM FORESTS REGRESSION
}

\author{
Ali Al-Yacoub \\ Intelligent Automation, Loughborough University \\ Holywell Building, Holywell Way \\ Loughborough, LE11 3QZ,UK \\ email: a.al-yacoub@lboro.ac.uk \\ Sara Sharifzadeh \\ Niels Lohse \\ Zahid Usman \\ Institute for Advanced Manufacturing and Engineering, Coventry University \\ Priory Street,Coventry \\ CV1 5FB,UK \\ Yee Mey Goh \\ Mike Jackson
}

\begin{abstract}
Haptics, as well as force and torque measurements, are increasingly gaining attention in the fields of kinesthetic learning and robot Learning from Demonstration (LfD). For such learning techniques, it is essential to obtain accurate force and torque measurements in order to enable accurate control. However, force and torque measurements using a 6-axis force and torque sensor mounted at the endeffector of an industrial robot are known to be corrupted due to the robots internal forces, gravity, un-modelled dynamics and nonlinear effects. This paper presents an evaluation of two techniques, SVR and Random Forests, to recover the external forces and accurately detect possible contact situations by estimating a robots internal forces. The performance of the learned models have been evaluated using different performance metrics and comparing them with respect to the features contained in the input space. Both SVR and Random Forests require low computational complexity without intensive training over the operational space under the given assumptions. In addition, these methods do not need data to be available online. The SVR and Random Forests models are experimentally validated using Motoman SDA10D dual-arm industrial robot controlled by Robot Operating System (ROS). The experiments showed that force and torque compensation based on Random Forests has outperformed Support Vector Regression.
\end{abstract}

\section{KEY WORDS}

force and torque compensation; Support Vector Regression (SVR); Random Forests (RF) regression and Industrial robots.

\section{Introduction}

Measuring contact forces when a manipulator carries out an assembly task is very important. However, the force/torque signals measured using 6-axis force torque sensor are corrupted due to the internal forces generated by the F/T sensor, the end-effector (gripper), workpiece and the unmodelled dynamics [12]. In learning applications, only the contact force patterns are important. The internal forces must be filtered out.

Noise and disturbances occurring at high frequencies can be greatly reduced using classical filters such as lowpass filter [19]. Furthermore, disturbances due to robot motion can not be omitted especially in high speed applications or when heavy robot tools are used. Approaches reported in the literature that address this problem generally use estimation methods based on dynamic models that consider the internal components of the sensor, gripper and workpiece [14].

The force/torque sensor are used to measures both external and internal forces. Measuring external forces is important in the context of force-based controlling or when a robot needs to interacts with the surrounding environment. The problem of estimating the external force/torque is generally defined as shown in Figure 1, where $\{\mathcal{O}\}$ is the robot base frame, $\left\{\mathcal{O}_{T}\right\}$ is the tool frame, $\left\{\mathcal{O}_{s}\right\}$ is the force/torque sensor frame, $\{\mathcal{O}\}$ is the end-effector frame and $r$ is the vector from the base frame $\{\mathcal{O}\}$ to the tool frame $\left\{\mathcal{O}_{T}\right\}$. The total effective force at the end effector can be calculated using Newton-Euler equations, as shown in Equation 1 and Equation 2.

$$
\sum \mathbf{F}=m \ddot{\mathbf{r}}=m \mathbf{g}+\mathbf{F}_{e}+\mathbf{F}_{s}
$$




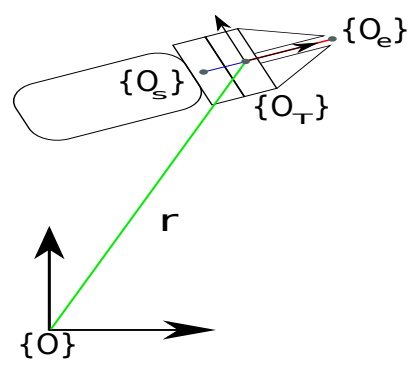

Figure 1. Dyanmic modelling of the force/torque measurement process.

$$
\sum \mathbf{T}=I \dot{\omega}+\omega \times I \omega=\mathbf{T}_{s}+r_{s} \times \mathbf{F}_{s}+\mathbf{T}_{e}+r_{e} \times \mathbf{F}_{e}
$$

Let $r$ be the position of the center of gravity of robot's tool (gripper+object), $\omega$ is the angular joints' velocities, $m$ is the total mass of the end effector, force/torque sensor and the workpiece, $I$ its' moment of inertia, $g$ the gravitational acceleration, $\mathbf{F}_{s}, \mathbf{T}_{s}$ and $\mathbf{F}_{e}, \mathbf{T}_{e}$ are the sensor and external generalized forces (force and torque), and $r_{e}$ and $r_{s}$ are the vectors from the center of the gravity to the sensor and the external force frames. Based on this dynamic model, many model-based estimation methods have been proposed. In [9] a Kalman filter was used to estimate the external forces and torques at the end effector. This model is suitable for high-speed applications. The main drawbacks are the high computational power and the model complexity. Another method proposed a joint based force torque estimation to estimate the contact force at the end effector using a Kalman filter [1]. This method requires an accurate model, a huge computational effort and a sensor fusion algorithm. Many researchers used a Kalman filter to estimate the contact force or external forces (such as object held by the end effector). In [11] contact forces and torques were estimated for multiple cooperative robots. Forces and torques for a fixable payload were estimated using a Kalman filter in [6]. The aforementioned research all use model-based methods that require high computational forces.

Alternatively, machine learning algorithms have been proposed to estimate forces and torques. For example, Locally Weighted Projection Regression (LWPR) was used to adjust a compliance matrix for computed torque controller [15]. However, this requires skillful tuning of the learning parameters. Also, Gaussian Process Regression (GPR) and LWPR were implemented to achieve an adaptive compliance control [16]. The results showed the advantage of using LWPR over GPR. In the same paper, Nguyen-Tuong and Peters introduced a Locally Gaussian Process (LGP) to learn a computed torque controller and compared it with SVR, Gaussian Mixture Regression (GMR) and LWPR. The results showed that LGP can achieve higher accuracy and requires lower computational forces compared to GMR and SVR. Also, LGP achieves higher accuracy compared to LWPR. Nonetheless, it requires higher computational force compared to LWPR.

Another supervised learning algorithm that has a good performance is RF. The RF was introduced by [3]. However, it is still not widely accepted in robotics applications. Caruana and Niculescu-Mizil presented a comparison amongst supervised learning algorithms, which found that RF is the second best algorithm after boosting [5]. Caruana et al. also compared the performance of the same supervised learning algorithms that was performed on a high dimensional data [4]. It was demonstrated work shows that RF has a steady performance over a wide range of dimensionalities. Andreas et al. proposed an active RF to overcome the active vision problem [7]. Active vision literature focuses on finding effective approaches to select observations with miner attention to the classification approaches. In that work, RF was used as a classification approach to identify clothes and grasping points. Also, active RF was used on-line to predict the grasping point positions that reduce the grasping error. Liarokapis et al. recorded muscular activity from human forearm and upper arm while an operator was performing a reach-tograsp activity where the object position was defined in $3-\mathrm{D}$ space [13]. In that work, RF was used to classify different Electromyography (EMG )signals for different reach-to-grasp strategies. Furthermore, Matteo et al. presented a comparison between model-based approaches and machine learning approaches to compensate internal force/torque for a humanoid robot [8]. The main result of that work was that the performance of learning algorithms exceed the performance of the analytical-models approach with regard to their prediction accuracy. Two machine learning approaches were implemented and compared, namely, Least-Square Super Vector Machine (LSSVM) and Neural-Network (NN). The LS-SVM converged more rapidly compared with NN. However, once they converged, eventually their performance was almost identical. The proposed data driven methods requires tuning, which is difficult due to the number of parameters that need to be tuned. Also, training data sometimes requires pre-processing and normalization to gain acceptable performance.

In this paper, RF regression has been utilised due to its simplicity, its ease to be tune and its' resistance to overfitting. Accordingly, it is believed that RF can overcome the limitations of the traditional robotics data driven models. Also, RF models will be compared with SVR which is one of the state-of-the-art machine learning algorithms, and have been well utilised in robotics context. SVR and RF, an off-line parametric regression methods, that have been used to estimate the internal forces of the end effector and workpiece for a pre-defined trajectory with limited speed. Both methods have been investigated for the force/torque compensation of an industrial robot. A 6-axis force/torque sensor was mounted between the end effector and the industrial robot. A pre-defined trajectory was executed by the 
industrial robot. Meanwhile, the sixth force/torque, joints variables, joint velocities, and acceleration were recorded. The collected data were used for training, cross-validation, and testing.

This paper is structured as follows: a brief introduction into RF is given in Section 2. Subsequently, Super Vector Regression is introduced in Section 3. After that, Section 4 introduces experimental setup and data collections and the learning process. This is followed by the discussion and results in Section 5. Finally, Section 6 concludes the paper and introduces future work.

\section{Random Forests (RF) Regression}

The RF developed by [3] combines bootstrapping (BAGGING) ([2]) and Decision Trees ([18]). The simplicity of the RF approach is the reason of its' popularity today. Moreover, it offers comparable performance to boosting - An RF is a large sets of decorrelated decision trees as shown in Equation 3. It contains $T$ number of trees where each tree $t$ is trained with a different bagged data using a selected of features that minimizes the error.

$$
h\left(x, \psi_{t}\right)_{t}=1, \ldots T
$$

where $x$ is an instance of an observation with $D$ number of random selected features, $\psi_{t}$ is a set of independent identically distributed random vectors (bagged data). Such that, the hypotheses $h\left(x, \psi_{t}\right)$ for tree $t$ takes a numerical value. The nature of those vectors specifies the tree construction. Where each tree predicts a numerical value based on a given observation $x$. The output values are numerical and it is assumed that the training set is independently emerging from a distribution of random vectors. RF capture complex interactions in data, it is relatively robust to noise and outliers and is claimed to be resistant to over-fitting [3]. The reason behind RF robustness against over-fitting the fact that it relies on averaging of large number of estimators (tress). Some of the main advantages of the RF technique are the fact that they run efficiently on large databases and are able to handle large set of input variables without variable deletion. Moreover, RF expose nonlinearity and have low generalization error.

Industrial robots are highly nonlinear systems and require precise modeling to achieve an accepted accuracy. However, modeling requires a deep understanding of the dynamical system (multi-objects dynamics) and it heavily relies on many assumptions. In contrast, data-driven models can achieve higher accuracy with less effort and adequate data. In this context, RF seems to be a promising machine learning model that can capture nonlinear models with high accuracy even with the presence of noise and disturbances. In this work, the input cases (features) considered as $(x \in\{q,[q, \dot{q}],[q, \dot{q}, \ddot{q}]\}))$ in order to study the models performances with different input features. As the output is limited to a single scalar value, a separate model has to be trained for each output dimension. In another word, 6 different models need to be trained for each of the force and torque components.

\section{Support vector Regression}

SVR aims to identify a decision boundary (hyperplane in high dimensional feature space) that fits the training data [10]. Equation 4 illustrates the model captured using SVR.

$$
f(x)=\mathbf{w}^{T} \mathbf{\Psi}(\mathbf{x})+b
$$

Where $\boldsymbol{\Psi}(\mathbf{x})$ is the projection of the input $x$ into the feature space, $\mathbf{w}$ and $b$ indicate the weights and the bias of the hyperplane, respectively. The weight vector $\mathbf{w}$ and the the bias $b$ are chosen to optimize the cost function which is shown in Equation 5.

$$
\min _{w} \frac{1}{2}\|\mathbf{w}\|^{2}+C \sum_{i=1}^{m} \mathcal{Q}\left(y_{i}-f\left(x_{i}\right)\right)
$$

Where $\mathcal{Q}(z)=\max \{0,|z|-\varepsilon\}$ is the $\varepsilon$ loss function (does not penalize errors less than $\varepsilon \geq 0$ ), $C$ is a regularisation factor that controls the relative weighting between the twin goals of optimising $|\mathbf{w}|^{2}$ and of assuring that most training data have a margin at least $\varepsilon$. Based on this, the Lagrangian can be formed as shown in Equation 6.

$$
\begin{aligned}
& \mathfrak{L}(w, b, \zeta, \alpha, r)=\frac{1}{2} w^{T} w+C \sum_{i=1}^{m} \zeta_{i} \\
& -\sum_{i=1}^{m} \alpha_{i}\left[y^{(i)}\left(x^{T} w+b\right)-1+\zeta_{i}\right]-\sum_{i=1}^{m} r_{i} \zeta_{i}
\end{aligned}
$$

Where $\alpha_{i}$ 's and $r_{i}$ 's are the Lagrangian multipliers associated with each sample. The dual can be solved by setting the derivative to zero. The result is given in Equation 7.

$$
\begin{aligned}
& \min _{\alpha} W(\alpha)=\sum_{i=1}^{m} \alpha-\frac{1}{2} \sum_{i, j=1}^{m} y^{(i)} y^{(j)} \alpha_{i} \alpha_{j}\left\langle x^{(i)}, x^{(j)}\right\rangle \\
& \text { s.t. } 0 \leq \alpha_{i} \leq C, i=1, \ldots, m \\
& \sum_{i, j=1}^{m} \alpha_{i} y^{(i)}=0
\end{aligned}
$$

The performance of SVR models strongly depends on the selection of both $C$, the kernel function and number of features. Hence, three sets of features have been used to train the models. The first one only contained the robot joint variables $q$. The second data set contained the joint variables and it's first derivatives ( $q$ and $\dot{q})$, while the third data set contained the joint variables and it's first and second derivative $(q, \underline{\dot{q}}$ and $\underline{\ddot{q}})$. 


\section{Experiment Setup}

The experimental setup consists of a 6-axis force/torque sensor, Motoman SDA10D dual arm robot, and one PC workstation. The Motoman SDA10A, shown in Figure 2 (A), is a dual-arm robot with 15 degrees of freedom (7 DoF per arm 1 DoF for the body). Each arm can lift a payload of $10 \mathrm{~kg}$. The PC workstation runs Ubuntu with Robot Operating System (ROS) ${ }^{1}$. The PC workstation is connected to the robot controller and the Force/torques sensor. The PC workstation can be used to collect data (training and testing) and to control the robot manipulator during task execution. The collected data include robot joint variables, end-effector Cartesian position, and Force/torque data.

The 6-axis force/torque sensor is adapted to collect internal forces during robot execution (one arm) of a predefined trajectory without any external loads. This predefined trajectory tries to cover a sub-space of the robot workspace. The collected data will be used to train an SVR and an RF model that map the joints information into the internal force/torque information that need to be compensated.

Regarding the training procedure, the five-fold crossvalidation method was used to select models parameters. The RF and SVR Models were tuned using grid search cross-validation from the Sklearn library [17]. The RF Regression models were tuned with the following parameters: \{number of trees: [100:100:2000], Maximum number of features in node: [2,21], Minimum number of samples at leaf: $[2,52,10]\}$, to select optimal parameters that maximize the performance. While SVR models were tuned with the following parameters: kernel:[ Radial Base Function (RBF),linear, poly, sigmoid ], C: $[1,10, \ldots, 1 \mathrm{e} 5]$ and : $[0.001, \ldots, 100]$ to select optimal hyperplane parameters. the variability, multiple rounds of cross-validation have been performed using different partitions. The validation results were averaged over the rounds. In the context of force compensation, each tree $t$ in the RF predicts internal force/torque signals and the final prediction is the average of all tree predictions.

An individual model is trained for each force and torque components using different numbers of features. Figure 2 (B) shows three different sets of training data, the outputs are the internal forces and torques. However, each training data set has a different number of features, i.e. the first set contains 7 features $(\underline{q})$, the second set contains 14 features ( $q$ and $\dot{q}$ ) and the third data set contains 21 features $(\underline{q}, \underline{\dot{q}}$ and $\underline{\tilde{q}})$. This has been done to observe the performance of the RF and SVR with different numbers of features.

For each data set 12 models have been trained using RF and SVR (6 each). Each model has been evaluated individually using unseen data. The prediction qualities of the trained models have been measured using their Mean Square Error (MSE), Normalized Mean Square Error (NMSE) and Variance Score. NMSE has been calculated for all 6 output dimensions using Equation 8. The

${ }^{1}$ ROS-Industrial (Hydro)

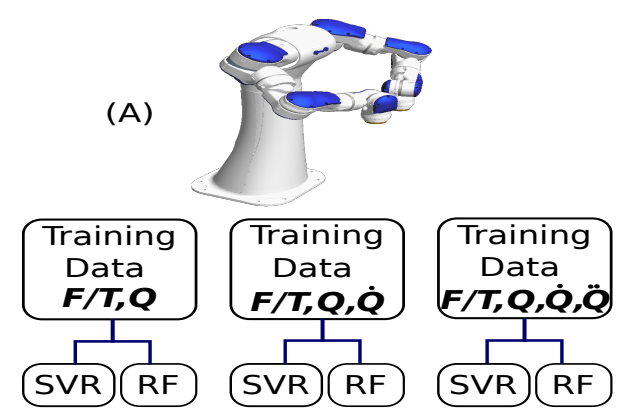

(B)

Figure 2. (A) SDA10D Motoman Dual arm robot. (B) Training data with different number of features.

NMSE is calculated over all 6 output dimensions. For the SVR method, the inputs were rescaled to a range between $[-1,+1]$ based on the maximum and minimum values found in each input dimension of the training set.

$$
\mathrm{NMSE}=N \frac{\sum_{i}^{N}\left(p^{(i)}-y^{(i)}\right)}{\sum_{i}^{N} p^{(i)} \sum_{i}^{N} y^{(i)}}
$$

Another important metric that can evaluate how will the prediction values are matching the true value can be measured using the explained variance score that can be estimated as shown in Equation (9).

$$
\operatorname{Var}(\hat{y}, y)=1-\frac{\operatorname{Var}\{y-\hat{y}\}}{\operatorname{Var}\{y\}}
$$

\section{Results and Discussion}

The methods introduced in the previous sections have been experimentally evaluated on data set collected for a predefined robot trajectory. The joint positions have been recorded from the robot controlled. The joints velocities and accelerations have been numerically differentiated. The three force components $\left(F_{x}, F_{y}\right.$ and $\left.F_{z}\right)$ and the three torque components $\left(T_{x}, T_{y}\right.$ and $\left.T_{z}\right)$ have been recorded directly from the sensor. The complete data set of 13,745 samples has been split into inputs for both training and testing. Each joint variable had a distribution as shown in Table 1.

As stated before, both machine learning approaches were utilised to train models as follow: firstly, only based on joints' variables $\left(q_{i}, i \in[1,7]\right)$, secondly using joints' variables and velocities and finally using joints variables, velocities, and accelerations. The performance of the trained models was measured using NMSE as illustrated in Figure 3, and MSE as shown in Figure 4. Based on these results, the RF clearly outperforms the SVR for all $\mathrm{F} / \mathrm{T}$ components. However, the errors are very high for the $Z$ components $\left(F_{z}\right.$ and $T_{z}$ ). The reason why RF and SVR did not capture the $F_{Z}$ and $T_{z}$ components precisely is that 
Table 1. Joints variable statistics

\begin{tabular}{c|ccccccc} 
Joints & $q_{1}$ & $q_{2}$ & $q_{3}$ & $q_{4}$ & $q_{5}$ & $q_{6}$ & $q_{7}$ \\
\hline $\max$ & $-18.48^{\circ}$ & $-1.80^{\circ}$ & $179.00^{\circ}$ & $-15.6^{\circ}$ & $-95.30^{\circ}$ & $100.65^{\circ}$ & $65.3^{\circ}$ \\
\hline $\min$ & $-132.70^{\circ}$ & $-103.30^{\circ}$ & $50.50^{\circ}$ & $-78.00^{\circ}$ & $-116.37^{\circ}$ & $71.10^{\circ}$ & $28.10^{\circ}$ \\
\hline$\mu$ & $-88.98^{\circ}$ & $-52.75^{\circ}$ & $131.41^{\circ}$ & $-52.24^{\circ}$ & $-109.63^{\circ}$ & $87.40^{\circ}$ & $48.98^{\circ}$
\end{tabular}

the pre-defined trajectory did not contain enough variations in the $Z$ axis. Based on this trajectory the robot will mostly move in a $X-Y$ plain. This means that the data collect can be captured the force and torques on the $X$ and $Y$ axes but, capturing $F_{z}$ and $T_{z}$ requires more variations in the $Z$ axis. Table 3 depicts the pre-defined trajectory's statistics in the Cartesian space $(X-Y-Z)$. Accordingly, the variations of the force in the $Z$ axis is lower than the variations in $X$ and $Y$ axes. Table 2 shows the mean and the stander deviation of the F/T components. This table shows that the smallest range of variation will occur on the $Z$ axis.

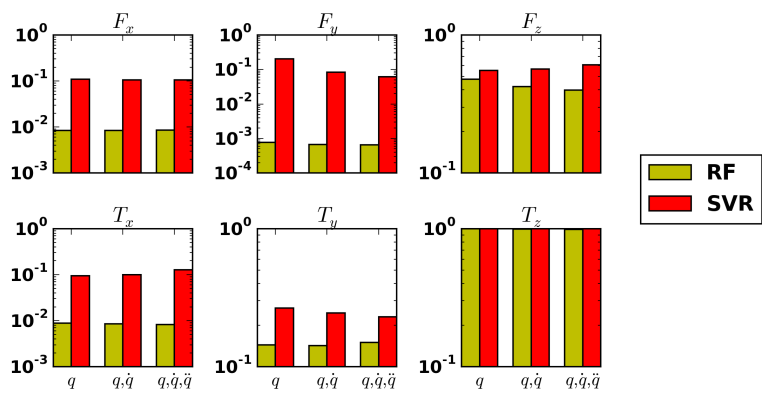

Figure 3. NMSE for RF models and SVR models.

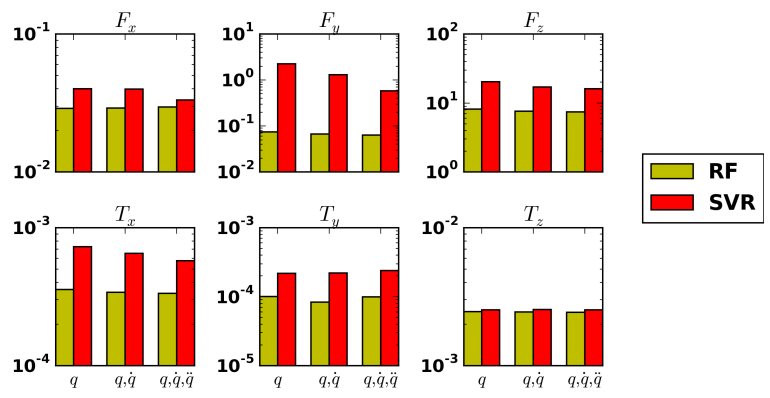

Figure 4. MSE for RF models and SVR models.

Figure 5 depicts the variance score of the $F_{x}, F_{y}$ and $F_{z}$ models trained using RF and SVR . Apparently, the variance square of the prediction error from the trained models using SVR and RF for $F_{z}$ is around 0.5 . Also, by including more features (joints' velocity and acceleration) the variance score of the RF model is increasing. In contrast, the variance score of SVR model is decreasing when including more features. Almost the same trend can be seen for the torque $T_{z}$ as shown in Figure 6.

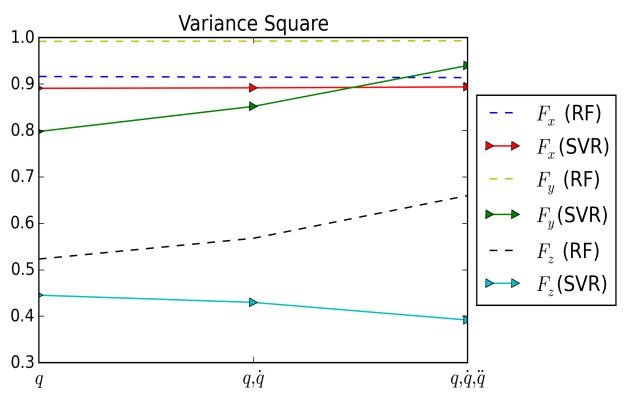

Figure 5. Variance score of the forces' models using RF and SVR.

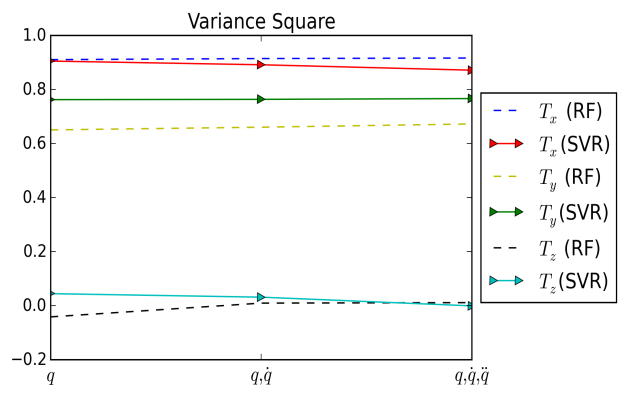

Figure 6. Variance score of the torques' models using RF and SVR .

Given the relatively low velocities and accelerations of the robot, the forces and torques are mainly generated due to gravity in relation to the current configuration of the robot (joints variables). Therefore, in RF case, it is constructive to train models only based on joints variables. However, include joints' velocities and acceleration can provide SVR models with more representative data that can be separable using kernels. Another advantage of the RF over SVR is that it can rank features based on their importance and it relies on the most important features for regression. This is the reason for the RF performance not

Table 3. Trajectory variation on $X, Y$ and $Z$ axis.

\begin{tabular}{cccc} 
& $X$ & $Y$ & $Z$ \\
\hline Maximum & 772.0 & -237.0 & -100.0 \\
\hline Minimum & 248.0 & -628.0 & -178.0 \\
\hline Average & 578.0 & -455.0 & -132.0
\end{tabular}


Table 2. Force/torque statistics

\begin{tabular}{c|cccccc} 
Joints & $F_{x}$ & $F_{y}$ & $F_{z}$ & $T_{x}$ & $T_{y}$ & $T_{z}$ \\
\hline $\max$ & 19.08 & 24.20 & 17.58 & -0.2 & -2.27 & 0.860 \\
\hline $\min$ & 15.97 & 13.08 & -4.02 & -0.064 & -2.20 & 0.610 \\
\hline$\mu$ & 17.40 & 20.06 & 6.85 & 0.04 & -2.24 & 0.741
\end{tabular}

being improved when more features are included. Figure 7 shows the important features for the $F_{x}$ model using all of the features $(\underline{q}, \dot{q}$ and $\ddot{q})$. This figure indicates that the RF regression model relies mostly on the joint variables to predict the undesired forces in the $X$ direction. Also, Figure 8 illustrates the importance of the features for the trained RF model using only the joint variables $(q)$. Clearly, both RF models use the same important features. This explains the unchanged performance of the RF when more features are included.

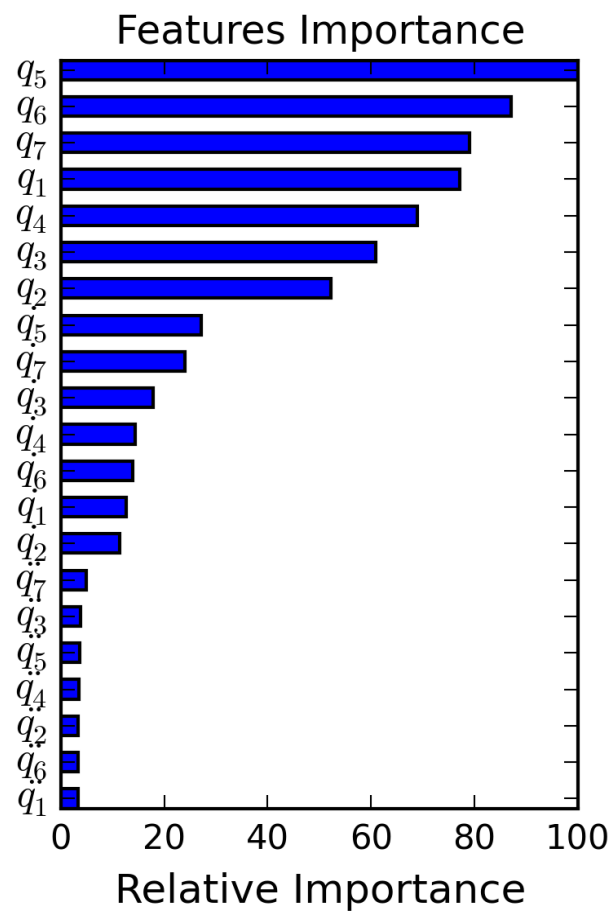

Figure 7. Important features for $F_{x}$ RF model using joint variables, velocities and accelerations

\section{Conclusion}

In this paper, two different approaches for compensating undesired force/torque features from a 6 -axis $\mathrm{F} / \mathrm{T}$ sensor mounted at the end effector of an industrial robot have been compared. Both approaches attempt to capture the physical behavior of the industrial robot based on data collected during a pre-defied experiment.

The variables of the models have been selected using

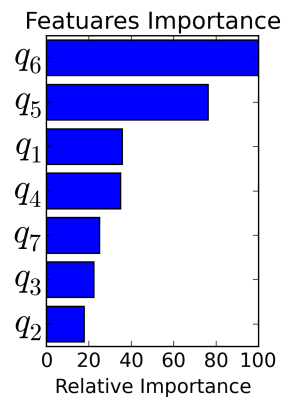

Figure 8. Important features for $F_{x} \mathrm{RF}$ model using joint variables, velocities and accelerations

cross-validation for both RF and SVR. Three sets of input features have been used for both approaches. The first one contained only the joints variables $\underline{q}$ while the second one contained the joints variables $\underline{q}$ and joints velocity $\underline{\dot{q}}$. The third set contained variables $q$, joints' velocities $\dot{q}$ acceleration $\underline{\underline{q}}$. Both regression methods have been trained using the same data and evaluated using unseen data. The NMSE, MSE, and variance score have been used as performance metrics for the derived models. The results indicated that including the joint velocities and accelerations did not enhance the performance of the RF models performance, while it improves the accuracy of the SVR models. In general, the RF models outperform the SVR even with noisy and corrupted data.

Even with noisy data on the $Z$ components the variance score shows that the RF can capture the forces and torques with acceptable performance compared to the Support Vector Machine. This paper shows a positive potential of utilising RF in robotics application. In the future work, pre-defined trajectories that cover more operational space should be utilised to better capture the robot dynamics and kinematics. Furthermore, the RF regression could be modified by weighting individual trees and features in order to improve the overall accuracy.

\section{acknowledgment}

The authors acknowledge support from the EPSRC Centre for Innovative Manufacturing in Intelligent Automation, in undertaking this research work under grant reference number EP/IO33467/1 


\section{References}

[1] D. Bi, Y.F. Li, S.K. Tso, and G.L. Wang. Friction modeling and compensation for haptic display based on support vector machine. Industrial Electronics, IEEE Transactions on, 51(2):491-500, April 2004.

[2] Leo Breiman. Bagging predictors. Machine Learning, 24(2):123-140, 1996.

[3] Leo Breiman. Random forests. Machine Learning, 45(1):5-32, 2001.

[4] Rich Caruana, Nikos Karampatziakis, and Ainur Yessenalina. An empirical evaluation of supervised learning in high dimensions. In Proceedings of the 25th International Conference on Machine Learning, ICML '08, pages 96-103, New York, NY, USA, 2008. ACM.

[5] Rich Caruana and Alexandru Niculescu-Mizil. An empirical comparison of supervised learning algorithms. In Proceedings of the 23 rd International Conference on Machine Learning, ICML '06, pages 161168, New York, NY, USA, 2006. ACM.

[6] C. Mavroids Avroids D. Economou, C. Lee and I. Antoniadis. Robust vibration suppression in flexible payloads carried by robot manipulators using digital filtering of joint trajectories. In Proceedings of the 2nd International Symposium on Robotics and Automation, 2000.

[7] Andreas Doumanoglou, Tae-Kyun Kim, Xiaowei Zhao, and Sotiris Malassiotis. Active random forests: An application to autonomous unfolding of clothes. In Computer Vision-ECCV 2014, pages 644-658. Springer, 2014.

[8] Matteo Fumagalli, Arjan Gijsberts, Serena Ivaldi, Lorenzo Jamone, Giorgio Metta, Lorenzo Natale, Francesco Nori, and Giulio Sandini. From Motor Learning to Interaction Learning in Robots, chapter Learning to Exploit Proximal Force Sensing: A Comparison Approach, pages 149-167. Springer Berlin Heidelberg, Berlin, Heidelberg, 2010.

[9] J.G. Garcia, A. Robertsson, J.G. Ortega, and R. Johansson. Generalized contact force estimator for a robot manipulator. In Robotics and Automation, 2006. ICRA 2006. Proceedings 2006 IEEE International Conference on, pages 4019-4024, May 2006.

[10] T. Hastie, R. Tibshirani, and J. Friedman. The Elements of Statistical Learning: Data Mining, Inference, and Prediction, Second Edition. Springer Series in Statistics. Springer, 2009.

[11] Manish Kumar and Devendra P. Garg. Sensor-based estimation and control of forces and moments in multiple cooperative. ASME JOURNAL OF DYNAMIC SYSTEMS, MEASUREMENT, AND CONTROL., 126:276-283, 2004.

[12] Achim Schweikard Lars Richter, Ralf Bruder. Calibration of force/torque and acceleration for an independent safety layer in medical robotic systems. Cureus medical journal, 59:1-14, 2012.

[13] M. V. Liarokapis, P. K. Artemiadis, P. T. Katsiaris, K. J. Kyriakopoulos, and E. S. Manolakos. Learning human reach-to-grasp strategies: Towards emg-based control of robotic arm-hand systems. In Robotics and Automation (ICRA), 2012 IEEE International Conference on, pages 2287-2292, May 2012.

[14] P. Lischinsky, C. Canudas-de Wit, and G. Morel. Friction compensation for an industrial hydraulic robot. Control Systems, IEEE, 19:25-32, Feb 1999.

[15] Duy Nguyen-Tuong, Matthias Seeger, and Jan Peters. Computed torque control with nonparametric regression models. In American Control Conference, 2008, pages 212-217. IEEE, 2008.

[16] Duy Nguyen-Tuong, Matthias Seeger, and Jan Peters. Model learning with local gaussian process regression. volume 23, pages 2015-2034. Taylor \& Francis, 2009.

[17] F. Pedregosa, G. Varoquaux, A. Gramfort, V. Michel, B. Thirion, O. Grisel, M. Blondel, P. Prettenhofer, R. Weiss, V. Dubourg, J. Vanderplas, A. Passos, D. Cournapeau, M. Brucher, M. Perrot, and E. Duchesnay. Scikit-learn: Machine learning in Python. Journal of Machine Learning Research, 12:28252830, 2011.

[18] J. R. Quinlan. Induction of decision trees. Mach. Learn., 1(1):81-106, March 1986.

[19] Baoyuan Wu, Qingquan Yan, Jianfei Luo, and Zhongcheng Wu. Signal processing and application of six-axis force/torque sensor integrated in humanoid robot foot. Journal of Signal Processing Systems, 74(2):263-271, 2014. 